\title{
Adaptação de um Pulverizador de Tração Humana para APLICAÇÃo LOCALIZADA DE HERBICIDAS ${ }^{1}$
}

\author{
Adaptation of a Manual Spray for Herbicide Application at Specific Site \\ VILIOTTI, C.A. ${ }^{2}$, VIEIRA, L.B. ${ }^{3}$, FERREIRA, L.R. ${ }^{4}$, DIAS, G.P. ${ }^{5}$ e RINALDI, P.C.N. ${ }^{6}$
}

\begin{abstract}
RESUMO - Objetivou-se com este trabalho adaptar um pulverizador de tração humana para aplicação localizada de herbicidas. Para o desenvolvimento do circuito eletrônico, foi elaborado um programa computacional em linguagem Assembly, com informações sobre a variação da resistência elétrica no LDR (Resistor Dependente de Luz) referente à radiação global incidente e refletida para quatro tipos de superficies diferentes (plantas dicotiledôneas, plantas monocotiledôneas, palhada de trigo e solo). Concluiu-se que é possivel e economicamente viável a utilização do sensor LDR para detecção de plantas daninhas.
\end{abstract}

Palavras-chave: agricultura de precisão, planta daninha, sensor.

\begin{abstract}
The objective of this work was to adapt a manual spray for herbicide application at specific site. For the development of the electronic circuit, a computer program in Assembly language was elaborated, with information on the variation of the electric resistance in LDR (Light Dependent Resistance), referring to the incident and reflected global radiation for four types of different surfaces (dicotyledonous plants, monocotyledonous plants, wheat straw and soil). It was concluded that it is possible and economically viable to utilize the LDR sensor to detect weeds.
\end{abstract}

Keywords: precision agriculture, weeds, sensor.

\section{INTRODUÇÃO}

A busca do aumento da produção no campo resultou na mecanização e na agricultura em grande escala. Como consequência, as grandes áreas de cultivo passaram a ser visualizadas como glebas homogêneas, levando ao conceito da necessidade média para a aplicação dos insumos. Assim, a mesma formulação e/ou quantidade de fertilizante é utilizada para toda a área, atendendo apenas às necessidades médias e não considerando, dessa forma, as necessidades específicas de cada parte da área (Tschiedel \& Ferreira, 2002).
Com a finalidade de atender às exigências específicas de cada parcela da área, surgiu o conceito de agricultura de precisão, que, segundo Molin (2001), é um elenco de tecnologias e procedimentos utilizados para que as lavouras e o sistema de produção sejam otimizados.

Para diminuir a quantidade de herbicida aplicado, várias pesquisas estão sendo realizadas utilizando a reflexão difusa da luz pelas plantas daninhas em tempo real, a fim de identificá-las no campo, usando como sensores ópticos câmeras de vídeo acopladas a computadores e software apropriado (Crowe $\&$ Delwiche, 1996; Tian, 1999).

1 Recebido para publicação em 19.3.2008 e na forma revisada em 18.2.2009.

2 Engo-Agrícola, Professor Adjunto da Universidade Federal do Ceará - UFC, Ceará-CE, <viliotti@ufc.br>; ${ }^{3}$ Engō-Agrícola, Professor Associado do Departamento de Engenharia Agrícola da Universidade Federal de Viçosa - DEA/UFV, 36570-000 ViçosaMG, <lbaiao@ufv.br>; ${ }^{4}$ Engo-Agrônomo, Professor Associado do Departamento de Fitotecnia - DFT/UFV, <lroberto@ufv.br>; ${ }^{5}$ Engọ-Agrônomo, Professor Adjunto do Departamento de Engenharia Agrícola - DEA/UFV, <gpdias@ufv.br>; ${ }^{6}$ Engạ-Agrícola, Doutoranda em Mecanização Agrícola - DEA/UFV, < pcnrinaldi@yahoo.com.br>. 
Os sensores ópticos (resistor dependente de luz - LDR) são dispositivos semicondutores, cuja resistência varia com a intensidade de radiação eletromagnética incidente, aumentando sua condutividade quando expostos à radiação. Hooper et al. (1976), estudando o efeito da luz refletida pelas plantas daninhas no LDR, detectaram que, quando a luz refletida incidia sobre os sensores, estes comandavam um circuito amplificador que registrava em um circuito contador uma intensidade associada ao número de plantas daninhas presentes entre as linhas da cultura; a eficiência do circuito foi de $99 \%$ no controle de plantas daninhas. Já Haggar et al. (1983) evidenciaram um desempenho de $90 \%$ no controle de plantas daninhas.

Este trabalho de pesquisa teve como objetivos desenvolver um circuito eletrônico de baixo custo para detecção da presença de plantas daninhas e controle da vazão de herbicidas, de modo que a pulverização fosse realizada somente na presença das plantas daninhas; e adaptar o sistema a um pulverizador de tração humana, tipo ciclojet.

\section{MATERIAL E MÉTODOS}

O projeto, a construção e os testes para verificar o funcionamento do protótipo foram conduzidos no campus da Universidade Federal de Viçosa.

O condicionamento de saída do LDR foi realizado com dois circuitos divisores de tensão (Figura 1): um para a radiação global incidente e outro para a refletida.

Considerando o cálculo dos circuitos divisores de tensão (equação 1), ao ocorrer uma variação na intensidade da radiação global incidente ou refletida, ocorrerá variação na resistência do LDR (R1) e na saída, proporcionalmente.

$$
\text { Vsaída }=\frac{R 2}{R 2+R 1} * \text { Ventrada }
$$

Para controle dos processos lógicos, medidas de tensão de saída dos circuitos condicionadores e decisão, abertura e fechamento da válvula solenóide, foi utilizado o microcontrolador PIC 16F871.
O programa computacional a ser executado pelo microcontrolador foi desenvolvido em linguagem Assembly, utilizando o sistema de programação MPLAB IDE for Windows/16 versão 5.30.00, que é uma ferramenta integrada, uma vez que, no mesmo ambiente de trabalho, possui o gerenciamento de projetos, a compilação, a simulação, a emulação e a gravação do microcontrolador.

Foram adaptados, em um pulverizador de tração humana (Figura 2), duas barras (uma para fixação dos sensores e outra para as válvulas solenóides), um regulador de pressão para manter a vazão de calda constante, o protótipo do circuito eletrônico e uma bateria para alimentação do sistema eletrônico e para a válvula solenóide.

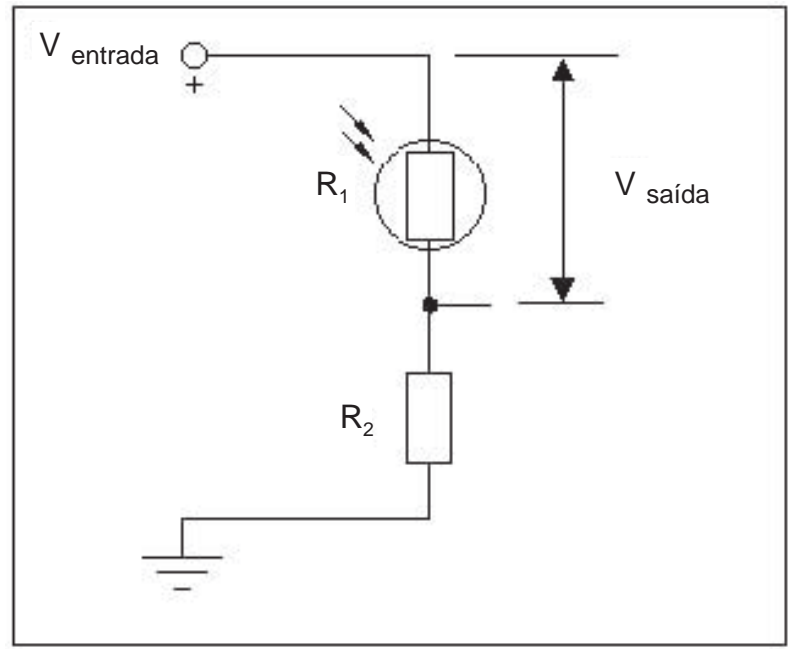

Figura 1 - Circuito divisor de tensão.

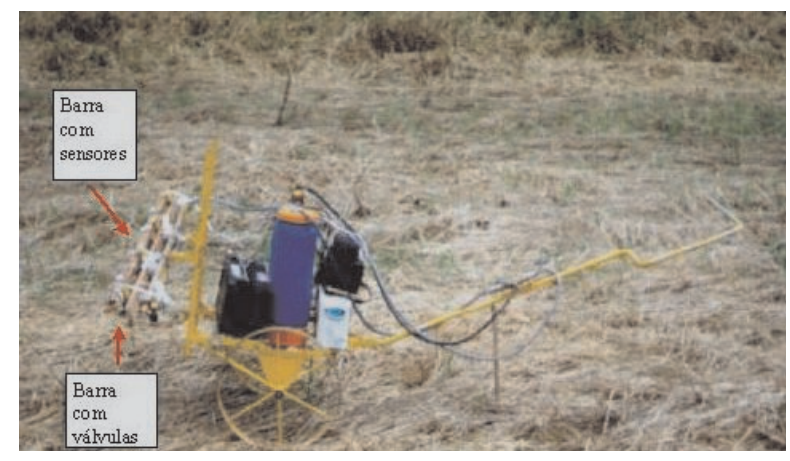

Figura 2 - Adaptação do pulverizador de tração humana tipo ciclojet. 
A válvula solenóide (Figura 3), marca comercial Transoni, adaptada para o desenvolvimento do protótipo foi escolhida previamente, por ser de fácil aquisição, possuir uma entrada simples e uma saída com diâmetro de $13,5 \mathrm{~mm}$ e apresentar pressão de trabalho entre 0,5 e $8,0 \mathrm{kgf} \mathrm{cm}^{-2}$, com tensão de alimentação de $127 / 220 \mathrm{~V}$, vida útil mínima de 50.000 ciclos e baixo custo de aquisição.

Pelo fato de a válvula solenóide ser de tensão alternada (127 Vca), foi necessária a elaboração de um circuito conversor de tensão contínua (12 Vcc).

Para a aferição do sistema eletrônico, o pulverizador foi deslocado em uma área (Figura 4), dividida em três parcelas: uma sem vegetação (palhada e solo) e duas com vegetação, sendo uma com predominância da população de picão-preto (dicotiledônea), com $210 \mathrm{~g} \mathrm{~m}^{-2}$ de matéria seca, e a outra com predominância de tiririca (monocotiledônea), com $140 \mathrm{~g} \mathrm{~m}^{-2}$ de matéria seca. Para obter esses tipos de vegetação e a área sem vegetação, foi realizada uma capina manual. O teste foi repetido 35 vezes, e dois observadores marcavam o número de vezes que a válvula foi aberta.

Para confirmação do resultado foi utilizado o herbicida paraquat na dose de $0,4 \mathrm{~kg} \mathrm{ha}^{-1} \mathrm{e}$ volume de calda de $85 \mathrm{~L} \mathrm{ha}^{-1}$, para dessecação de contato.

O pulverizador foi deslocado com velocidade de $1,1 \mathrm{~m} \mathrm{~s}^{-1}$, pressão de trabalho do bico hidráulico de $180 \mathrm{kPa}$, ponta de pulverização de plano 80015, faixa de pulverização igual a $0,90 \mathrm{~m}$, volume de calda de $85 \mathrm{~L} \mathrm{ha}^{-1}$, altura da barra de 0,60 $\mathrm{m}$ em relação ao solo, estando as plantas daninhas com até $0,20 \mathrm{~m}$ de altura, e espaçamento de $2 \mathrm{~m}$ entre as faixas pulverizadas e de $0,50 \mathrm{~m}$ entre os bicos na barra.

\section{RESULTADOS E DISCUSSÃO}

A equação escolhida para ser introduzida no programa computacional foi a da palhada de trigo (Figura 5), em que o LDR - Superior $(\Omega)$ recebe a radiação global incidente e o LDR Refletida $(\Omega)$ recebe a radiação global refletida. Essa escolha foi feita porque a palhada reflete mais luz sobre o sensor do que as folhas e menos que o solo.

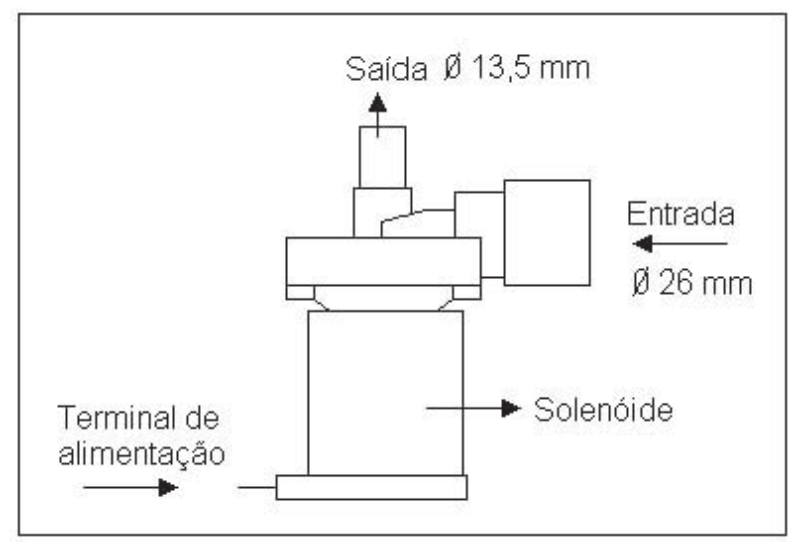

Figura 3 - Válvula solenóide (fora de escala).

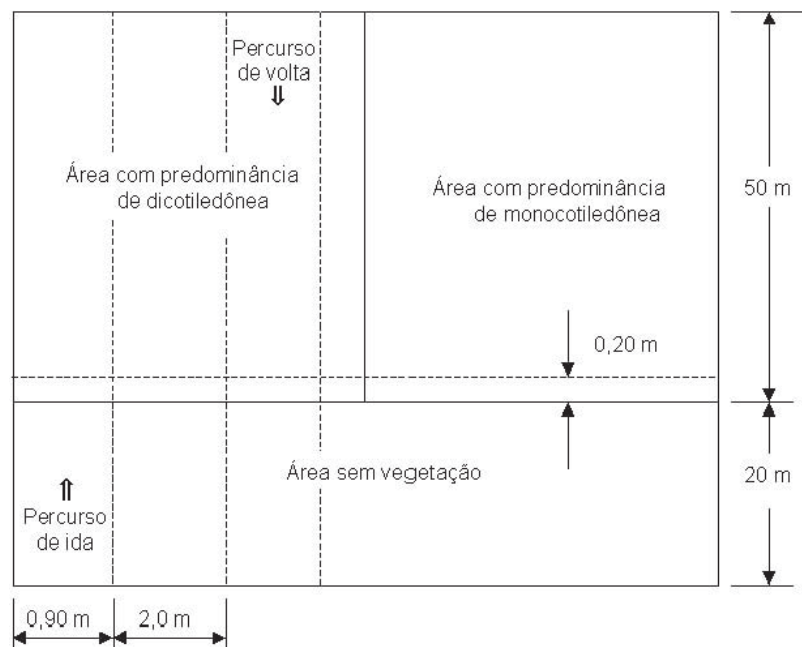

Figura 4 - Esquema da área experimental.

Com o aumento da intensidade de luz refletida no LDR, um número maior de elétrons na sua estrutura tem seu nivel de energia aumentado, devido à aquisição de energia entregue pelos fótons. O resultado é o aumento de elétrons livres disponíveis para a condução elétrica, ocorrendo diminuição da resistência elétrica do LDR.

O circuito eletrônico desenvolvido para o protótipo, apresentado na Figura 6, é constituído por regulador de tensão (A); circuito oscilador, $4 \mathrm{MHz}(\mathrm{B})$; circuito divisor de tensão (C); circuito de controle de saída - foi utilizado um relé de $6 \mathrm{~V}$ (D); e um circuito microcontrolador (E). Os valores foram: $\mathrm{C} 1$ e C2 $=100$ $\mathrm{nF} ; \mathrm{C} 3$ e C4 = $18 \mathrm{pF} ; \mathrm{R} 1$ e R2 = $10 \mathrm{k} \Omega ; \mathrm{R} 3=1 \Omega$; $\mathrm{X} 1=4 \mathrm{MHz} ; \mathrm{e} \mathrm{T} 1=\mathrm{BC} 547$.

Planta Daninha, Viçosa-MG, v. 27, n. 1, p. 205-209, 2009 
A corrente consumida pelo protótipo foi de 0,011 A, sem o acionamento do relé, e 0,052 A, com o acionamento, com uma alimentação de $5 \mathrm{~V}$. Na alimentação do protótipo foi utilizada uma bateria de $12 \mathrm{~V} 120 \mathrm{AH}$.

Para o funcionamento da válvula, foi construído um circuito conversor de corrente contínua para alternada; a corrente consumida pela válvula foi de $0,097 \mathrm{~A}$, com alimentação de $97 \mathrm{~V}$ e potência de dissipação de 9,4 W.

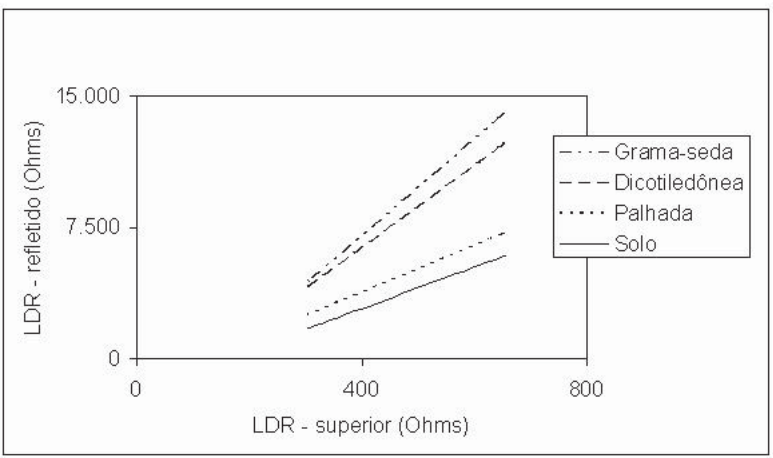

Figura 5 - Resistência do sensor de luz refletida, em função da resistência do sensor de luz incidente
A válvula foi submetida ao teste na bancada: em um intervalo de 1 segundo ela permanecia aberta, e 0,5 segundo, fechada. Durante duas horas, não ocorreu anormalidade em seu funcionamento.

Nos testes de campo, a válvula permaneceu fechada toda vez que o pulverizador se encontrava em uma área sem vegetação; ao entrar em uma área com vegetação depois de 0,08 segundo, tempo de resposta do sistema, a válvula era acionada pelo circuito eletrônico, permanecendo aberta durante o tempo em que o pulverizador permanecia sobre essa área, sendo observado um controle de $100 \%$ das áreas infectadas com plantas daninhas nessas condições.

De acordo com as condições em que o experimento foi realizado, conclui-se que: o sistema eletrônico desenvolvido para detecção da presença de plantas daninhas obteve sucesso no campo; o sensor LDR mostrou que é possivel e economicamente viável a sua utilização para detecção de plantas daninhas; e o custo total do protótipo foi da ordem de R $\$ 50,00$ (US\$1,00 $=\mathrm{R} \$ 1,70 \mathrm{em} \mathrm{24/6/2008).}$ O microcontrolador utilizado (16F871), já obsoleto, pode ser

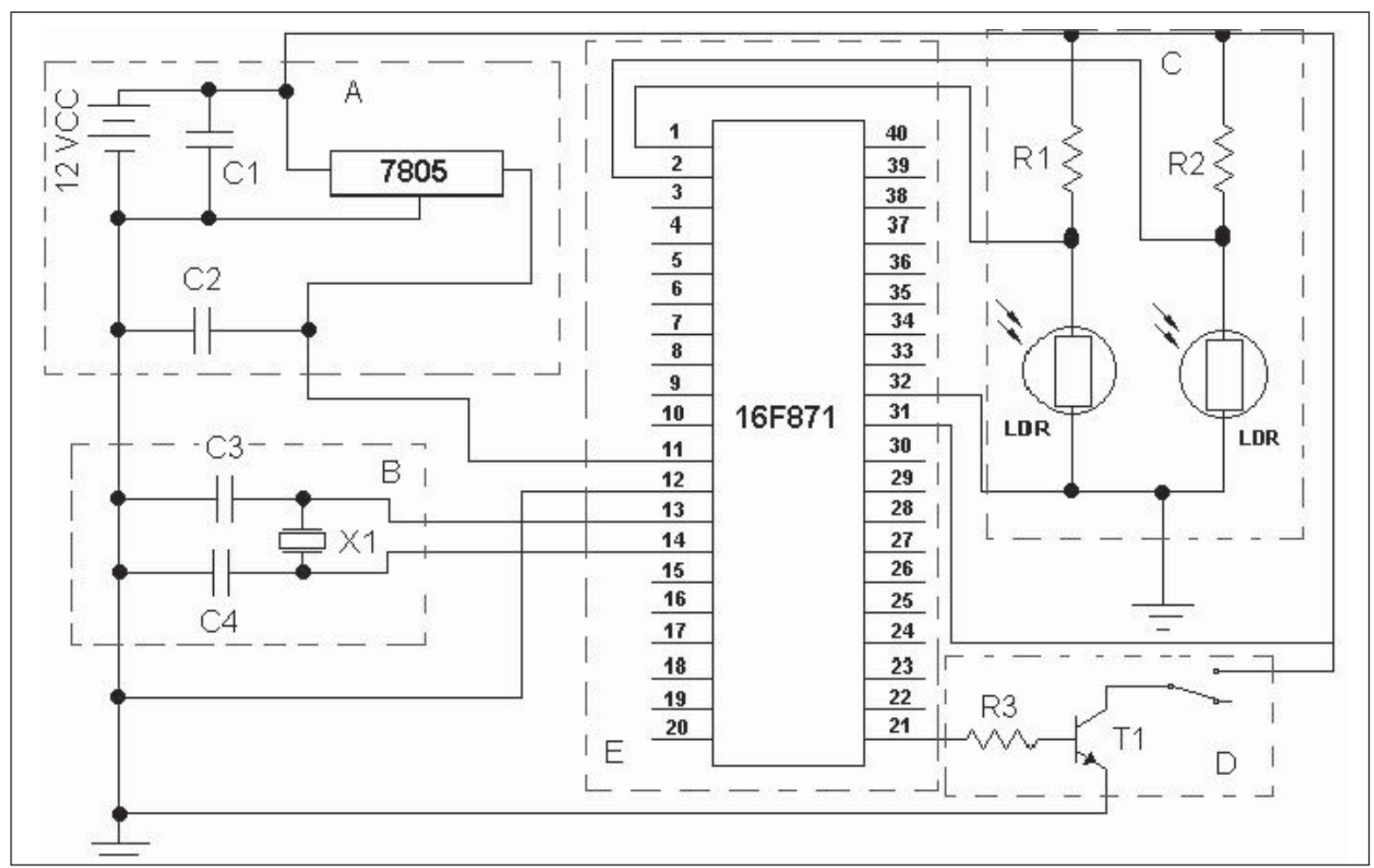

Figura 6 - Circuito eletrônico para detecção de plantas daninhas e controle da aplicação de herbicidas. 
substituído por outro da mesma família (16F877) ou de outras familias.

\section{LITERATURA CITADA}

CROWE, T. G.; DELWICHE, M. J. Real-time defect detection in fruit - part 1: design concepts and development of prototype hardware. Trans. Am. Sci. Agric. Eng., v. 39, n. 2, p. 2299-2308, 1996.

HAGGAR, R. J.; STENT, C. J.; ISSAC, S. A prototype hand-held patch sprayer for killing weeds, activated by spectral difrerenoes in crop weed canopies. J. Agric. Eng. Res., v. 28, p. 349-358, 1983.
HOOPER, A. W.; HARRIES, G. O.; AMBLER, B. A photoeletric sensor for distinguishing between plant material and soil. J. Agric. Eng. Res., v. 21,n. 1, p. 145-155, 1976.

MOLIN, J. P. Agricultura de precisão: o gerenciamento da variabilidade. Piracicaba: Ediçao do Autor, 2001. 83 p.

TIAN, L.Precision chemical application system (smart sprayer project). 31/05/1999 Disponível em: (http:// www.age. uiuc. edu/faculty/lft/sprayer.htm).

TSCHIEDEL, M.; FERREIRA, M. F.Introdução à agricultura de precisão: conceitos e vantagens. Ci. Rural, v. 32, n. 1, p. 159-163, 2002. 